To appear in the Journal of Korean Statistical Society (2012):

\title{
Wavelet Based Estimation for the Derivative of a Density by Block Thresholding under Random Censorship
}

Esmaeel Shirazi, Department of Statistics, Ferdowsi University, Mashhad, 917551159, Iran (esm.shirazi@stu-mail.um.ac.ir)

Yogendra P. Chaubey, Department of Mathematics and Statistics, Concordia University, Montréal, QC H3G 1M8 (chaubey@alcor.concordia.ca)

Hassan Doosti , Department of Mahematics, Tarbiat Moalem University, Tehran, Iran(hassandoosti1353@yahoo.com)

H.A. Nirumand, Department of Statistics, Ferdowsi University, Mashhad, 91755-1159, Iran

Abstract: We consider wavelet based method for estimating derivatives of a density via block thresholding when the data obtained are randomly right censored. The proposed method is analogous to that of Hall and Patil (1995) for density estimation in the complete data case that has been extended recently by $\mathrm{Li}(2003,2008)$. We find bounds for the $L_{2}$-loss over a large range of Besov function classes for the resulting estimators. The results of Hall and Patil (1995), Prakasa Rao (1996) and Li $(2003,2008)$ are obtained as special cases and the performance of proposed estimator is investigated by numerical study.

Keywords: Adaptive estimation; Block thresholding; Censored data; Nonparametric estimator of derivative of a density; Rates of convergence.

\section{Introduction}

In various applications, estimation of a density and its derivatives may be required, for example, for the evaluation of modes and inflection points, for estimation of the derivatives of a regression function and for evaluation of the density scores. There are 
many excellent texts that are now available on the subject dealing with theoretical aspects and applications. In this context the reader may be referred to the texts by Silverman (1986), Devroye (1987), Wand and Jones (1995), concentrating more on kernel methods (Rosenblatt (1956) and Parzen (1962)) and its variants. For a more theoretical treatment of the general topic of nonparametric functional estimation, one may consult for Prakasa Rao (1983). There are two excellent texts originating from the discipline of econometrics that provide an excellent review of various nonparametric methods for estimation of a density and its derivative (see Pagan and Ullah (1999) and Li and Racine (2006)). In most of these applications the kernel method is more popular and as a result many software packages include this method as a member of their toolbox, for example, one may see Wand and Ripley (2009) for the details of the KernSmooth package in $\mathrm{R}$ that is becoming a powerful tool for statisticians lately.

Estimation of derivatives of densities was featured in Bhattacharya (1967) in estimating the Fisher's information from a sample of i.i.d. observations and this theme was carried out in Singh (1977) highlighting many other applications using the estimators of density derivatives. Härdle et al. (1990) discussed the issue of the band width choice, specifically for density derivatives.

Initially, after the advent of the kernel method most of the investigations were with complete data until the paper by McNichols and Padgett (1984) that presented a modified kernel estimator for the density based on the right censored data. Padget and McNichols (1984) provided the status of density estimation for censored data until 1984. Further, Diehl and Stute (1988) considered hazard rate estimation using the kernel method. For a recent review on smooth functional estimation under censored data the reader may be referred to Chaubey et al. (2007).

This is the subject matter of the present paper where the data typically represent the survival times that are incomplete in some way due to the presence of a number of events which potentially censor the event of interest. Withdrawals from a clinical trial, deaths unrelated to the disease under study, individuals still alive at the end of a follow-up study, and so on, are examples of censoring issues.

Currently, wavelet based methods for estimation of density and derivatives are becoming increasingly popular as an alternative to the usual kernel method. These methods offer fast computations and easy updating in addition to being easily adapted to the design (Delouille et al. (2001)), and specific smoothness (Donoho and Johnstone (1995)). Donoho et al. [DJKP](1995) proposed wavelet based methods for density estimation for the i.i.d. data which were subsequently adapted to estimation of derivatives by Prakasa Rao (1996). Hall and Patil (1995) used a modification of DJKP estimator adapting to a specific smoothness parameter and obtained an asymptotic formula for the mean integrated squared error of the corresponding density estimator. Further work in this context may be referred, specifically for density estimation by Hall et al. (1999), Antoniadis et al. (1999), Cai (1999) and Li (2003) 
and for nonparametric regression function estimation by Dabrowska (1995) and Cai (2002). Recently, Li (2002, 2003) has extended Hall and Patil's (1995) analysis to hazard rate and density estimation under right random censorship model, respectively. We may mention here that Patil (1997) also considered estimation of hazard rate based on orthogonal wavelets, however, under uncensored data. In this paper, we follow a similar plan as in $\mathrm{Li}(2003,2008)$ for analyzing the block thresholding wavelet estimator for the $d$-th $(d \geq 0)$ derivative of a density using censored data. Our analysis parallels to that of $\mathrm{Li}$ (2008) dealing with density estimation in providing an upper bound on $L^{2}$-loss for the resulting estimator. Naturally, for $d=0$, we get the result in $\mathrm{Li}$ (2008) that optimal convergence rates for density estimation are achieved over a large range of Besov function classes.

The organization of the paper is as follows. In Section 2 we describe some preliminary notions about the wavelet system and Besov spaces. In Section 3, the form of block thresholding wavelet estimator for the $d$-th derivative of a density is given along with the main result that gives an upper bound on the $L_{2}-$ loss for the estimator. In section 4 , we study the performance of various wavelet threshold estimators. Details of the steps in the sketch of the proof are relegated to the Appendix.

\section{Preliminaries on Wavelets and Besov Spaces}

Here we provide a brief introduction to the wavelet system and Besov spaces that have become essential to the statistical literature. For the details of the theory and applications of wavelets, the reader may refer to the excellent text by Vidakovic (1999) or to the excellent survey by Antoniadis (2007). For properties of the Besov spaces, the reader is referred to Meyer (1992) and Triebel (1992) (cf. Leblanc (1996), Härdle et al. (1998)).

\subsection{Wavelet System}

A wavelet system is composed of an infinite collection of functions that are obtained by dilation and translation of two basic functions $\phi($.$) and \psi($.$) called the scaling$ function and mother wavelet, respectively. The function $\phi$ is assumed to satisfy

$$
\int_{-\infty}^{\infty} \phi(x) d x=1
$$

and is obtained as the solution from the equation

$$
\phi(x)=\sum_{k=-\infty}^{\infty} C_{k} \phi(2 x-k),
$$


for a given sequence of constants $\left\{C_{k}\right\}$, and the function $\psi(x)$ is given by

$$
\psi(x)=\sum_{k=-\infty}^{\infty}(-1)^{k} C_{-k+1} \phi(2 x-k) .
$$

Define

$$
\phi_{i, j}(x)=2^{i / 2} \phi\left(2^{i} x-j\right), \quad-\infty<i, j<\infty
$$

and

$$
\psi_{i, j}(x)=2^{j / 2} \phi\left(2^{i} x-j\right), \quad-\infty<i, j<\infty .
$$

Suppose that the coefficients $\left\{C_{k}\right\}$ satisfy

$$
\begin{aligned}
\sum_{k=-\infty}^{\infty} C_{k} C_{k+2 l} & =2 \text { if } l=0 \\
& =0 \text { if } l \neq 0 .
\end{aligned}
$$

It is known (cf. Daubechies (1992)) that under some additional conditions on $\phi($.$) ,$ the collection $\left\{\psi_{i, j} ;-\infty<j<\infty,-\infty<k<\infty\right\}$ forms an orthonormal basis for $L^{2}(\mathbb{R})$, and $\left\{\phi_{i_{0}, j} ;-\infty<j<\infty\right\}$ constitute an orthonormal basis for $L^{2}(\mathbb{R})$, for every fixed $i_{0} \in \mathbb{Z}$, as well.

Definition 2.1. The scaling function $\phi$ is said to be $r$-regular for an integer $r \geq 1$, if for every nonnegative integer $\ell \leq r$, the $\ell$-th derivative of $\phi(),. \phi^{\ell}($.$) is such that$ for any integer $k \geq 1$,

$$
\left|\phi^{\ell}(x)\right| \leq c_{k}(1+|x|)^{-k},-\infty<x<\infty .
$$

for some $c_{k} \geq 0$ depending only on $k$.

Definition 2.2. A multiresolution analysis of $L^{2}(\mathbb{R})$ consists of an increasing sequence of closed spaces $\left\{V_{j}\right\}$ of $L^{2}(\mathbb{R})$ such that

(i) $\bigcap_{j=-\infty}^{\infty} V_{j}=\{0\}$;

(ii) $\bigcup_{j=-\infty}^{\infty} V_{j}=L^{2}(\mathbb{R})$;

(iii) there is a scaling function $\phi \in V_{0}$ such that $\{\phi(x-k),-\infty<k<\infty\}$ forms an orthonormal basis for $V_{0}$;

(iv) for all $h(.) \in L^{2}(\mathbb{R}),-\infty<k<\infty, h(x) \in V_{0} \Longrightarrow h(x-k) \in V_{0}$; and

(v) $h(x) \in V_{j} \Longrightarrow h(2 x) \in V_{j+1}$. 
Mallat (1989) has connected the multiresolution analysis to wavelet theory by showing that given any multiresolution analysis, it is possible to construct a function $\psi($.$) , (called the mother wavelet), such that for any fixed j,-\infty<j<\infty$, the family $\left\{\psi_{j, k}, j,-\infty<k<\infty\right\}$ constitutes a basis of the orthogonal complement $W_{j}$ of $V_{j}$ in $V_{j+1}$ so that $\left\{\psi_{j, k},-\infty<j, k<\infty\right\}$ is an orthonormal basis of $L^{2}(\mathbb{R})$ (cf. Daubechies (1992)). The corresponding multiresolution analysis is said to be $r$-regular if the scaling function $\phi($.$) is so.$

Suppose that both the functions $\phi$ and $\psi$ belong the space of functions with $r$ continuous derivatives denoted by $\mathbf{C}^{r}$, for some $r \geq 1$, and have compact supports included in $[-L, L]$, for some $L>0$. It follows, from Corollary 5.5.2 in Daubechies (1988), that the mother wavelet $\psi$ is orthogonal to polynomials of degree $\leq$ r, i.e.

$$
\int \psi(x) x^{l} d x=0, \forall l=0,1, \ldots, r
$$

Any function $f \in L^{2}(\mathbb{R})$ can be expanded in the form (cf. Daubechies (1992)):

$$
\begin{aligned}
f(x) & =\sum_{j \in Z} a_{i_{0}, j} \phi_{i_{0}, j}(x)+\sum_{i \geq i_{0}} \sum_{j \in Z} b_{i, j} \psi_{i, j}(x) \\
& =\mathcal{P}_{i_{0}} f(x)+\sum_{i \geq i_{0}} \mathcal{D}_{i} f(x)
\end{aligned}
$$

for any integer $i_{0} \in \mathbb{Z}$. The so called wavelet coefficients $a_{i_{0}, j}$ and $b_{i, j}$ are given by

$$
a_{i_{0}, j}=\int f(x) \phi_{i_{0}, j}(x) d x
$$

and

$$
b_{i, j}=\int f(x) \psi_{i, j}(x) d x
$$

respectively.

For the later analysis, we choose $i_{0}=0$ and use the notation

$$
a_{j} \equiv a_{i_{0}, j} \text { and } \phi_{j} \equiv \phi_{i_{0}, j}
$$

\subsection{Besov Spaces}

Besov spaces are normed spaces defined for weakly-differentiable functions belonging to $L^{2}(\mathbb{R})$. We present the following definition of a weakly differentiable function $f$ from Härdle et al. (1998). 
Definition 2.3. Let $f \in L^{2}(\mathbb{R})$ be an integrable function on every bounded interval. It is said to be weakly differentiable if there exists a function $g$ defined on the real line which is integrable on every bounded interval such that

$$
\int_{x}^{y} g(u) d u=f(y)-f(x)
$$

The function $g$ is defined almost everywhere and is called the weak derivative of $f$.

Then it is known that for any $\phi \in D(\mathbb{R})$,

$$
\int_{-\infty}^{\infty} f(u) \phi^{\prime}(u) d u=-\int_{-\infty}^{\infty} g(u) \phi(u) d u
$$

where $D(\mathbb{R})$ denotes the space of infinitely differentiable functions, on the real line, with compact support.

Definition 2.4. Let $1 \leq p \leq \infty$ and $m \geq 0$ be an integer. A function $f \in L_{p}(\mathbb{R})$ belongs to the Sobolev space $W_{p}^{m}(\mathbb{R})$, if it is $m$-times weakly-differentiable and the $m$-th weak derivative $f^{(m)} \in L_{P}(\mathbb{R})$. The space $W_{p}^{m}(\mathbb{R})$ is equipped with the norm $\|f\|_{W_{p}^{m}}$, called the Sobolev norm, where $\|f\|_{p}$ denotes the norm for $L_{p}(\mathbb{R})$.

Let $f \in L_{p}(\mathbb{R})$ for some $1 \leq p \leq \infty$. Let $\Delta_{h} f(x)=f(x+h)-f(x)$ and define $\Delta_{h}^{2} f=\Delta_{h}\left(\Delta_{h} f\right)$. For $t \geq 0$, let

$$
\omega_{p}^{1}(f, t)=\sup _{|h| \leq t}\left\|\Delta_{h} f\right\|_{p}
$$

and

$$
\omega_{p}^{2}(f, t)=\sup _{|h| \leq t}\left\|\Delta_{h}^{2} f\right\|_{p}
$$

Let $1 \leq q \leq \infty$. Suppose there exists a function $\epsilon(t)$ on $[0, \infty)$ such that $\|\epsilon\|_{p}^{*}<\infty$ where

$$
\begin{aligned}
\|\epsilon\|_{q}^{*} & =\left(\int_{0}^{\infty} t^{-1}|\epsilon(t)|^{q} d t\right)^{1 / q}, \text { if } 1 \leq q<\infty \\
& =\operatorname{ess} \sup _{t}|\epsilon(t)|, \text { if } q=\infty .
\end{aligned}
$$

Definition 2.5. Let $1 \leq p, q \leq \infty$ and $s=m+\alpha$ where $m \geq 0$ is an integer and $0<\alpha \leq 1$. The Besov space $B_{p, q}^{s}$ is the space of all functions $f$ such that $f \in W_{p}^{m}(\mathbb{R})$ and $\omega_{p}^{2}\left(f^{(m)}, t\right)=\epsilon(t) t^{\alpha}$ where $\|\epsilon\|_{q}^{*}<\infty$. 
The norm on the Besov space is induced by the corresponding Sobolev space that can be written as

$$
\|f\|_{B_{p, q}^{s}}=\left\|\mathcal{P}_{0} f\right\|_{p}+\left(\sum_{i \geq 0}\left(\left\|\mathcal{D}_{i} f\right\|_{p} 2^{i s}\right)^{q}\right)^{1 / q}
$$

where

$$
\mathcal{P}_{0} f=\sum_{j \in Z} a_{j} \phi_{j}
$$

represents the orthogonal projection of $f$ on the space spanned by functions $\phi_{j}, j \geq 0$ and

$$
\mathcal{D}_{i} f=\sum_{j \in Z} b_{i, j} \psi_{i, j}
$$

represents that on the space spanned by functions $\psi_{i, j}, j \in \mathbb{Z}$. Suppose that $f$ belongs to the Besov class (see Meyer (1992), §VI.10),

$$
F_{p, q}^{s}(M, L)=\left\{f \in B_{p, q}^{s},\|f\|_{B_{p, q}^{s}} \leq M, \operatorname{supp} f \subset[-L, L]\right\}
$$

for some $0 \leq s \leq r+1, p \geq 1$ and $q \geq 1$. Then in view of the representation (2.9), it can be shown (cf. Härdle et al. (1998)) that the function say $f \in B_{p, q}^{s}$ if and only if

$$
\left.\left\|a_{i_{0}, .}\right\|_{\ell_{p}}<\infty, \text { and } \sum_{i \geq i_{0}}\left(\left\|b_{i, .}\right\|_{\ell_{p}} 2^{i \sigma}\right)^{q}\right)^{1 / q}<\infty
$$

where $\sigma=s+(1 / 2)-(1 / p)$, and $\left\|\gamma_{j}, .\right\|_{\ell_{p}}$ represents the following norm for a double sequence $\left\{\gamma_{j, k}\right\}$ :

$$
\left\|\gamma_{i, .}\right\|_{\ell_{p}}=\left(\sum_{k \in Z} \gamma_{i, k}^{p}\right)^{1 / p}
$$

Let $\phi($.$) be the scaling function as defined earlier. Define$

$$
\theta_{\phi}(x)=\sum_{k=-\infty}^{\infty}|\phi(x-k)| .
$$

Suppose the following conditions hold:

(C1) The ess $\sup _{x} \theta_{\phi}(x)<\infty$ where

$$
\left.e s s \sup _{x} g(x)=\inf \{y: \lambda(\mid x: g(x)>y])=0\right\}
$$

and $\lambda$ is the Lebesgue measure on the real line. 
(C2) There exists a bounded nondecreasing function $\Phi($.$) such that |\Phi(u)| \leq \Phi(|u|)$ almost every where and

$$
\int_{0}^{\infty}|u|^{r} \Phi(|u|) d u<\infty
$$

for some integer $r \geq 0$.

Then the Besov norm can be written (here we take $i_{0}=0$ ) (cf. Härdle et al. (1998), p. 123) in terms of the wavelet coefficients:

$$
\|f\|_{B_{p, q}^{s}}=\left\|a_{0 .}\right\|_{p}+\left(\sum_{j \geq 0}\left(2^{j \sigma}\left\|b_{j,}\right\|_{p}\right)^{q}\right)^{1 / q} .
$$

\section{Block Thresholding Estimator of the $d$-th Deriva- tive of a Probability Density Function}

Let $\left\{X_{1}, X_{2}, \ldots\right\}$ and $\left\{Y_{1}, Y_{2}, \ldots\right\}$ be two sequences of random variables. The first sequence may be regarded as representing survival times (or failure times), having a common unknown distribution function $F($.$) and density function f($.$) and the second$ sequence as the censoring times as a result of random right censoring. The censoring times are assumed to be distributed according to a common distribution function $G($.$) . In this set up we may only observe$

$$
Z_{i}=\min \left(Y_{i}, X_{i}\right):=Y_{i} \wedge X_{i} \quad \text { and } \quad \delta_{i}=I\left(X_{i} \leq Y_{i}\right),
$$

where $I($.$) denotes the indicator function. In this random censorship model, we$ assume that the survival times $\left\{X_{i}\right\}$ are independent of the censoring times $\left\{Y_{i}\right\}$. Following the convention in the survival analysis literature, we assume that both $X_{i}$ and $Y_{i}$ are nonnegative random variables. In contrast to statistics for complete data, that are based on the sequence $\left\{X_{1}, X_{2}, \ldots\right\}$, the estimation for the censored data depends on the pairs $\left(Z_{1}, \delta_{1}\right),\left(Z_{2}, \delta_{2}\right), \ldots,\left(Z_{n}, \delta_{n}\right)$. For example the Kaplan-Meier estimators of the distribution functions $F$ and $G$ are defined, respectively, by

$$
\begin{aligned}
& \hat{F}_{n}(x)=1-\prod_{i=1}^{n}\left[1-\frac{\delta_{(i)}}{n-i+1}\right]^{I\left(Z_{(i)} \leq x\right)}, \\
& \hat{G}_{n}(x)=1-\prod_{i=1}^{n}\left[1-\frac{1-\delta_{(i)}}{n-i+1}\right]^{I\left(Z_{(i)} \leq x\right)},
\end{aligned}
$$

where $Z_{(1)} \leq Z_{(2)} \leq \ldots \leq Z_{(n)}$ denote the order statistics of $Z_{1}, Z_{2}, \ldots, Z_{n}$, and is the concomitant of $Z_{(i)}$, i.e., $\delta_{(m)}=\delta_{k}$ if $Z_{(m)}=Z_{k}$. Note that $\delta_{k} / n\left(1-\hat{G}\left(Z_{m}^{-}\right)\right)$is the jump of the Kaplan-Meier estimator $\hat{F}_{n}$ at $Z_{m}$. 
We are interested in estimating $f^{(d)}$, the $d$-th $(d \geq 0)$ derivative of $f$ based on $\left(Z_{i}, \delta_{i}\right), i=1,2, \ldots, n$. Note that the case $d=0$ refers to estimation of density itself. Let $T<\tau_{H}$ be a fixed constant, where $\tau_{H}=\inf \{x: H(x)=1\} \leq \infty$ is the least upper bound for the support of $H$, the distribution function of $Z_{1}$ and $f_{1}(x)=f(x) I(x \leq$ $T)$. We would like to estimate the $d$-th $(d \geq 0)$ derivative of $f$ over the interval $x \in(-\infty, T)$, or equivalently we estimate $f_{1}^{(d)}(x)$, for $x \in(-\infty, T)$. To motivate the estimator, we consider the wavelet expansion of $f_{1}$ as in Hall and Patil (1995) (see their $\S 2.2$ with $s=0)($ cf. $\operatorname{Li}(2008))$,

$$
\begin{aligned}
f_{1}(x) & =\sum_{j \in Z} a_{j} \phi_{j}(x)+\sum_{i \geq 0} \sum_{j \in Z} b_{i j} \psi_{i j}(x), \\
a_{j} & =\int f_{1}(x) \phi_{j}(x) d x, \quad b_{i j}=\int f_{1}(x) \psi_{i j}(x) d x .
\end{aligned}
$$

The non-linear wavelet estimator of $f_{1}$, as given below (see Li (2008), Eq (2.5)) may be motivated by the plug-in method resulting in:

$$
\hat{f}_{1}(x)=\sum_{j \in Z} \hat{a}_{j} \phi_{j}(x)+\sum_{i=0}^{\infty} \sum_{j \in Z} \hat{b}_{i j} I\left(\left|\hat{b}_{i j}\right|>\eta\right) \psi_{i j}(x),
$$

where $\eta>0$ is a "threshold" parameter ; the constants $\hat{a}_{j}$ and $\hat{b}_{i j}$ are defined as

$$
\begin{aligned}
\hat{a}_{j} & =\int I(x \leq T) \phi_{j}(x) d \hat{F}_{n}(x)=\frac{1}{n} \sum_{i=1}^{n} \frac{\delta_{i} I\left(Z_{i} \leq T\right) \phi_{j}\left(Z_{i}\right)}{1-\hat{G}\left(Z_{i}^{-}\right)}, \\
\hat{b}_{i j} & =\int I(x \leq T) \psi_{i j}(x) d \hat{F}_{n}(x)=\frac{1}{n} \sum_{i=1}^{n} \frac{\delta_{i} I\left(Z_{i} \leq T\right) \psi_{i j}\left(Z_{i}\right)}{1-\hat{G}\left(Z_{i}^{-}\right)},
\end{aligned}
$$

For estimators of the derivatives we use the generalized Fourier coefficients of $f_{1}^{(d)}$ (see $§ 2.5$ of Hall and Patil (1995)), namely,

$$
\begin{aligned}
\alpha_{j}^{(d)} & =\int f_{1}^{(d)} \phi_{j}=(-1)^{d} \int f(x) \phi^{(d)}(x-j) d x, \\
\beta_{i j}^{(d)} & =\int f_{1}^{(d)} \psi_{i j}=(-1)^{d} 2^{i(d+(1 / 2))} \int f(x) \psi^{(d)}\left(2^{i} x-j\right) d x .
\end{aligned}
$$

Using plug-in estimators of $\alpha_{j}^{(d)}$ and $\beta_{i j}^{(d)}$, the nonlinear wavelet thresholding estimator of $f_{1}^{(d)}$ is given by

$$
\tilde{f}_{1}^{(d)}(x)=\sum_{j \in Z} \hat{\alpha}_{j} \phi_{j}(x)+\sum_{i=0}^{\infty} \sum_{j \in Z} \hat{\beta}_{i j} I\left(\left|\hat{\beta}_{i j}\right|>\lambda\right) \psi_{i j}(x),
$$


where we have suppressed the superfix $(d)$ from the estimators of the wavelet coefficients, i.e.

$$
\begin{aligned}
\hat{\alpha}_{j} & =(-1)^{d} \int \phi_{j}^{(d)}(x) I(x \leq T) d \hat{F}_{n}(x) \\
& =(-1)^{d} \sum_{m=1}^{n} \frac{\delta_{m} I\left(Z_{m} \leq T\right) \phi_{j}^{(d)}\left(Z_{m}\right)}{n\left(1-\hat{G}_{n}\left(Z_{m}^{-}\right)\right)}, \\
\hat{\beta}_{i j} & =(-1)^{d} \int \psi_{i j}^{(d)}(x) I(x \leq T) d \hat{F}_{n}(x) \\
& =(-1)^{d} \sum_{m=1}^{n} \frac{\delta_{m} I\left(Z_{m} \leq T\right) \psi_{i j}^{(d)}\left(Z_{m}\right)}{n\left(1-\hat{G}_{n}\left(Z_{m}^{-}\right)\right)} .
\end{aligned}
$$

Note that $\delta_{m} / n\left(1-\hat{G}_{n}\left(Z_{m}^{-}\right)\right)$is the jump of the Kaplan-Meier estimator $\hat{F}_{n}$ at $Z_{m}$.

The above estimator in Eq. (3.5) is known as a 'hard-thresholding' wavelet estimator and may not achieve the optimal convergence rate (see Li (2008)) for wavelet density estimation. Hence a block thresholding method (see Cai (1999, 2002) and Chicken and Cai (2005) for details) as described in Li (2008) may be employed. This method provides the following form of the wavelet estimator of $f^{(d)}(x)$,

$$
\hat{f}_{1}^{(d)}(x)=\sum_{j \in Z} \hat{\alpha}_{j} \phi_{j}(x)+\sum_{i=0}^{R} \sum_{k \in \mathbb{Z}} \sum_{j \in \Gamma_{i k}} \hat{\beta}_{i j} \psi_{i j}(x) I\left(\hat{B}_{i k}>C n^{-1}\right),
$$

where $R$ is a smoothing parameter, $\Gamma_{i k}=\{j:(k-1) l+1 \leq j \leq k l\},-\infty<k<\infty$ represents consecutive, nonoverlapping blocks of length $l$ for each resolution $i$ and $\hat{B}_{i k}\left(=l^{-1} \sum_{j \in \Gamma_{i k}} \hat{\beta}_{i j}^{2}\right)$ represents the average estimated squared bias for the block $\Gamma_{i k}$. This may be presented in a simplified form as

$$
\hat{f}_{1}^{(d)}(x)=\sum_{j \in Z} \hat{\alpha}_{j} \phi_{j}(x)+\sum_{i=0}^{R} \sum_{k \in \mathbb{Z}} \hat{d}_{i k}(x) I\left(x \in J_{i k}\right) I\left(\hat{B}_{i k}>C n^{-1}\right),
$$

where $\hat{d}_{i k}(x)=\sum_{j \in \Gamma_{i k}} \hat{\beta}_{i j} \psi_{i j}(x)$ and

$$
J_{i k}=\bigcup_{j \in \Gamma_{i k}}\left\{x: \psi_{i j} \neq 0\right\}=\bigcup_{j \in \Gamma_{i k}}\left\{\operatorname{supp} \psi_{i j}\right\} .
$$

Our basic aim is to extend the results of Li (2008) that deals with density estimation to derivatives of compactly supported densities. For completeness we reproduce some of his notations that will be useful for further analysis. Define the reproducing wavelet kernel $K(x, y)=(-1)^{d} \sum_{j} \phi_{j}(x) \phi_{j}^{(d)}(y)$ (see Müller and Gasser (1979)). 
By self-similarity of multiresolution subspaces, $K_{i}(x, y)=2^{i(s+1)} K\left(2^{i} x, 2^{i} y\right), i=$ $0,1,2, \ldots$, is a reproducing kernel on the space spanned by functions $\phi_{j}, j \in \mathbb{Z}$. Now, we may define

$$
\begin{aligned}
& \mathcal{K}_{i} f^{(d)}(x)=\int K_{i}(x, y) f(y) d y, \\
& \mathcal{D}_{i} f^{(d)}(x)=\int D_{i}(x, y) f(y) d y
\end{aligned}
$$

where $D_{i}(x, y)=(-1)^{d} \sum_{j} \psi_{i j}(x) \psi_{i j}^{(d)}(y)$.In terms of the above notation wavelet expansion of $f_{1}^{(d)} \in L^{2}$, may be written as

$$
f_{1}^{(d)}(x)=\mathcal{K}_{0} f_{1}^{(d)}(x)+\sum_{i=0}^{\infty} \mathcal{D}_{i} f_{1}^{(d)}(x)
$$

where $\mathcal{K}_{0} f^{(d)}$ represents the orthogonal projection of $f^{(d)}$ on the space spanned by functions $\phi_{j}, j \in \mathbb{Z}$, and $\mathcal{D}_{i} f^{(d)}$ represents that on the space spanned by functions $\psi_{i j}, j \in \mathbb{Z}$.

Noting that $D_{i}(x, y)=K_{i+1}(x, y)-K_{i}(x, y)$ and there exists an integrable function $Q$ such that $|K(x, y)| \leq Q(x-y)$ for all $x, y$, which implies that for all integers $i$ and all $1 \leq p \leq \infty$,

$$
\left\|\mathcal{K}_{i} f^{(d)}\right\|_{p} \leq\|Q\|_{1}\|f\|_{p} \quad \text { and } \quad\left\|\mathcal{D}_{i} f^{(d)}\right\|_{p} \leq\|Q\|_{1}\|f\|_{p}
$$

We will also use the following estimators for $\mathcal{K}_{i} f^{(d)}(x)$ and $\mathcal{D}_{i} f^{(d)}(x)$ as suggested by Equations (3.10) and (3.11):

$$
\begin{aligned}
\hat{\mathcal{K}}_{i}^{(d)}(x) & =\frac{1}{n} \sum_{m=1}^{n} K_{i}\left(x, X_{m}\right), \\
\hat{\mathcal{D}}_{i}^{(d)}(x) & =\frac{1}{n} \sum_{m=1}^{n} D_{i}\left(x, X_{m}\right)
\end{aligned}
$$

In what follows we consider wavelets $\phi$ and $\psi$ as those in Cohen, Daubechies and Vial (1993), i.e. we assume that $\phi_{j}$ and $\psi_{i j}$ are compactly supported in $[-L, T]$ and form a complete orthonormal basis of $L^{2}(-L, T)$. Li (2008) considered a subset of densities in Besov class $B_{p, q}^{s}$ for $s>1 / p ; p, q \in[1, \infty)$. Our choice of the subset of the Besov class is different in that it depends on the order of the derivative to be estimated. For example, for $p \in[2, \infty)$, we assume that $s>\max (d, 1 / p)$. This obviously gives the same subset as in $\mathrm{Li}(2008)$ for $d=0$. Since, $F_{p, q}^{s}(M, L)$ is a subset of the space of bounded functions, we study the wavelet estimator for densities 
belonging to $F_{p, q}^{s}(M, L) \bigcap B_{\infty}(A)$ where $B_{\infty}(A)$ is the space of all densities $f$ such that $\|f\|_{\infty} \leq A$.

The following theorem extends Theorem 3.1 of $\mathrm{Li}$ (2008) for the wavelet estimators of the derivatives of densities over certain Besov spaces.

Theorem 3.1. Assume that the wavelets $\phi$ and $\psi$ are $(r+d)$-regular and have $d$ bounded derivatives. Let $\hat{f}_{1}^{(d)}$ be the block thresholding wavelet estimator (3.8) where the block length $l=\log n$, and $R=\left\lfloor\log _{2}\left(n l^{-2}\right)\right\rfloor$ and the thresholding constant $C$ is given as

$$
C=\frac{112.5 A\left(C_{2}\|Q\|_{2}+\sqrt{2} C_{1}^{-1 / 2}\|Q\|_{1}\right)^{2}}{([1-F(T)][1-G(T)])^{2}}
$$

with $C_{1}$ and $C_{2}$ being universal constants as given in Talagrand (1994) (see also Li (2008), pp 1535). Then there exists a constant $C_{0}$ such that for all $M, L \in(0, \infty)$ and $q \in[1, \infty]$ :

(i) For $p \in[2, \infty]$ and $s \geq \max (d, 1 / p)$,

$$
\sup _{\left.f^{(d)} \in F_{p, q}^{s}(M, L)\right) \cap B_{\infty}(A)} E \int\left(\hat{f}_{1}^{(d)}-f_{1}^{(d)}\right)^{2} \leq C_{0} n^{-2(s-d) /(1+2 s)},
$$

(ii) For $p \in[1,2)$ and $s \geq \max \left(\frac{(2 d+1)+(2-p) / 2}{p}, 1 / p\right)$,

$$
\sup _{\left.f^{(d)} \in F_{p, q}^{s}(M, L)\right) \cap B_{\infty}(A)} E \int\left(\hat{f}_{1}^{(d)}-f_{1}^{(d)}\right)^{2} \leq C_{0}\left(\log _{2} n\right)^{\frac{(2-p)(2 d+1)}{p(1+2 s)}} n^{-2(s-d) /(1+2 s)},
$$

Remark 3.1: This study can be considered as an extension from complete data to randomly right censored data. If we assume that there is no censoring, i.e., $G \equiv 0$ on $(-\infty, \infty)$, then $\delta_{i} \equiv 1$, for all $i=1,2, \ldots, n$ and we get the same result in Hall and Patil (1995), Prakasa Rao (1996) and Chaubey et al. (2006, 2008). Also, assuming $d=0$, we get the same result as in $\mathrm{Li}$ (2008) based on censored data.

Remark 3.2: Since the choice of block size $l$ and thresholding constant $C$ largely determines the performance of the resulting estimator, it is important to study in detail the effect of $l$ and $C$ on the properties of the estimator and derive the optimal $l$ and $C$ if such values exist. Between the two parameters $l$ and $C$, the block size $l$ is more important and plays a similar role as the bandwidth in the traditional kernel estimation. Here we can consider the block thresholding estimator in (3.8) with general block size $l=(\log n)^{s}$ with some $s \geq 0$, but based on Cai (2002), we know that to achieve the optimal global adaptivity, the block size must be at least of the 
order $O(\log n)$. On the other hand, to achieve the optimal local adaptivity, the block size must be no more than $O(\log n)$. Therefore no block thresholding estimator can achieve simultaneously the optimal global and local adaptivity if the block size is not of order $O(\log n)$.

\section{A Simulation Study}

In this section we study the performance of proposed estimator (3.9) and compare it with various other threshold wavelet estimators as available in the WaveLab package developed by Buckheit et al. (1995) at Stanford University. It may also be better to refer to Antoniadis et al. (2001) for a detailed description of the procedures. In the following two examples, we generate two sets of random variables and find the average norm criterion (ANorm) calculated for several thresholding estimators, including (3.9) for different sample sizes $n$. We show in detail the results for (ANorm) and the total number of replications $N=100$ and $d=1$; the ANorm criterion is defined as

$$
\text { ANorm }=\frac{1}{N} \sum_{l=1}^{N}\left(\sum_{i=1}^{n}\left(\hat{f}_{l}^{(d)}\left(x_{i}\right)-f_{l}^{(d)}\left(x_{i}\right)\right)^{2}\right)^{1 / 2} .
$$

In this simulation study, we used Daubechiess compactly supported wavelet Symmlet 4 (see Daubechies, 1992, p. 198) and Coiflet 2 (see Daubechies, 1992, p. 258), and primary resolution level $j_{0}=3$. The code was written in MATLAB environment using WaveLab software.

Example 1. Here we generate the random samples $X_{i}, 1 \leq i \leq n$ from a Beta distribution with parameters $\alpha=2, \beta=5$ along with the independent random sample $Y_{i}, 1 \leq i \leq n$ from the uniform distribution on the interval $(0,1)$. Table 1 , shows the values of ANorm for several wavelet estimators of derivative of beta density function in different sample size. Block thresholding estimator has better performance in comparing other wavelet estimators. When sample size increases the performance of other estimators come closer to the performance of our proposed estimator. Figure 1 shows the original derivative of $p d f$ of $X_{i}$ with black line, Block thresholding estimator with red line, hard thresholding estimator with blue line and linear estimator with dotted line respectively.

Example 2. For this example we consider the simulated data from an exponential family. We generate our sample, $X_{i}, 1 \leq i \leq n$ from exponential distribution 
Table 1: Computed values for ANorm and simulated standard errors for Example 1 for various sample sizes

\begin{tabular}{|l||c|c|c|c|}
\hline \multicolumn{1}{|c||}{ Estimation Methods } & \multicolumn{4}{c|}{ ANorm and (Simulated standard error) } \\
\cline { 2 - 5 } & $\mathrm{n}=256$ & $\mathrm{n}=512$ & $\mathrm{n}=1024$ & $\mathrm{n}=2048$ \\
\hline \hline Block Thresholding & $33.378(6.670)$ & $24.103(4.318)$ & $19.186(2.651)$ & $16.627(1.994)$ \\
\hline NeighBlock & $33.558(6.716)$ & $24.149(4.312)$ & $19.160(2.674)$ & $16.578(1.998)$ \\
\hline Minimax & $32.891(5.499)$ & $24.046(3.507)$ & $19.180(2.398)$ & $16.731(1.878)$ \\
\hline SureShrink & $39.544(5.204)$ & $28.454(3.893)$ & $21.895(2.579)$ & $18.378(1.893)$ \\
\hline Hard threshold & $39.858(5.609)$ & $27.842(4.158)$ & $21.374(2.687)$ & $17.769(2.051)$ \\
\hline Linear & $45.061(4.684)$ & $32.446(2.934)$ & $24.824(2.381)$ & $20.485(1.803)$ \\
\hline
\end{tabular}

with mean 1 . The sample, $Y_{i}, 1 \leq i \leq n$ is generated from exponential density with mean 5. Table 2 and Figure 2 give similar results as in Example 1.

Table 2: Computed values for ANorm and simulated standard errors for Example 1 for various sample sizes

\begin{tabular}{|l||l|l|l|l|}
\hline \multicolumn{1}{|c||}{ Estimation Methods } & \multicolumn{4}{c|}{ ANorm and (Simulated standard error) } \\
\cline { 2 - 5 } & $\mathrm{n}=256$ & $\mathrm{n}=512$ & $\mathrm{n}=1024$ & $\mathrm{n}=2048$ \\
\hline \hline Block Thresholding & $22.786(10.100)$ & $16.043(5.525)$ & $11.455(4.221)$ & $9.814(2.206)$ \\
\hline NeighBlock & $23.117(10.193)$ & $16.202(5.658)$ & $11.611(4.241)$ & $9.928(2.181)$ \\
\hline Minimax & $25.873(8.366)$ & $18.419(4.483)$ & $13.193(3.502)$ & $10.863(1.100)$ \\
\hline SureShrink & $30.733(11.608)$ & $21.032(6.865)$ & $13.893(5.087)$ & $11.873(3.249)$ \\
\hline Hard threshold & $31.721(10.529)$ & $21.557(6.290)$ & $14.3871(4.900)$ & $11.555(2.936)$ \\
\hline Linear & $44.839(7.547)$ & $32.707(4.126)$ & $23.687(3.458)$ & $17.871(2.100)$ \\
\hline
\end{tabular}

In Table 2, the simulation results show that the average norm for the block thresholding estimator is usually slightly smaller than those for other thresholding estimators.

Remark 4.1: Similar numerical study for density function $p d f$ estimation was carried out in Li (2008) where ANorm values are smaller than those in our study. However, we should note that the values in $\mathrm{Li}$ (2008) are for estimation of the probability density functions whereas in our case these are for the estimation of its derivative. Note that the rate of convergence for estimating the $p d f$ is not the same as the rate of convergence for estimating its derivative. 
Figure 1: Derivative estimation for Beta density

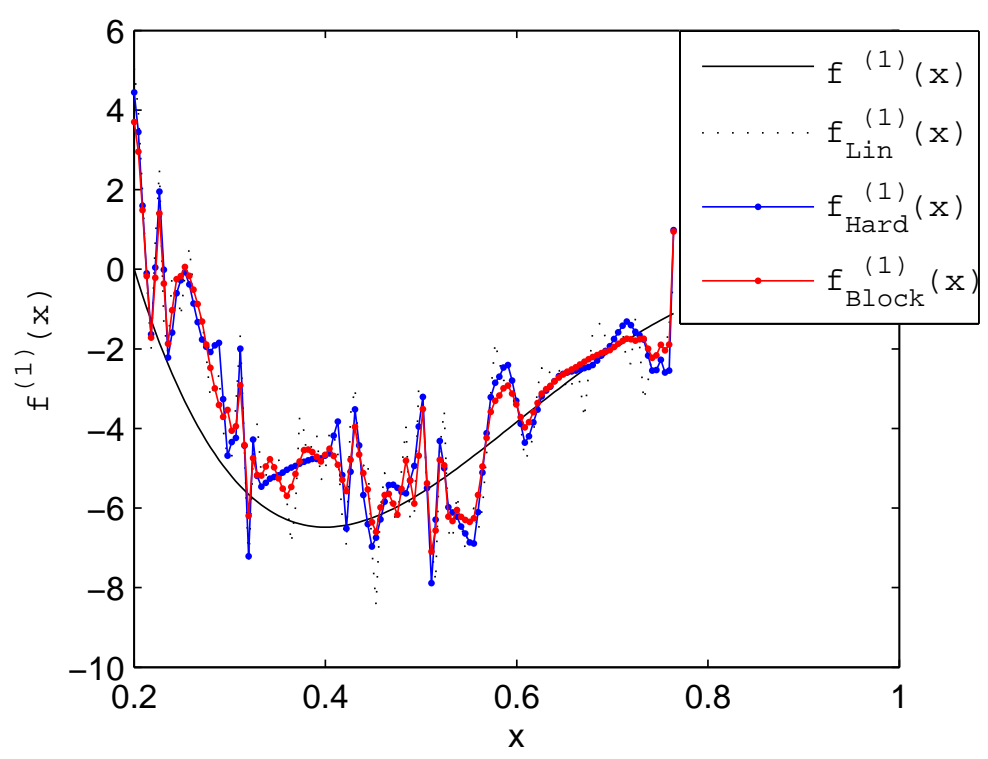

Figure 2: Derivative estimation for exponential density

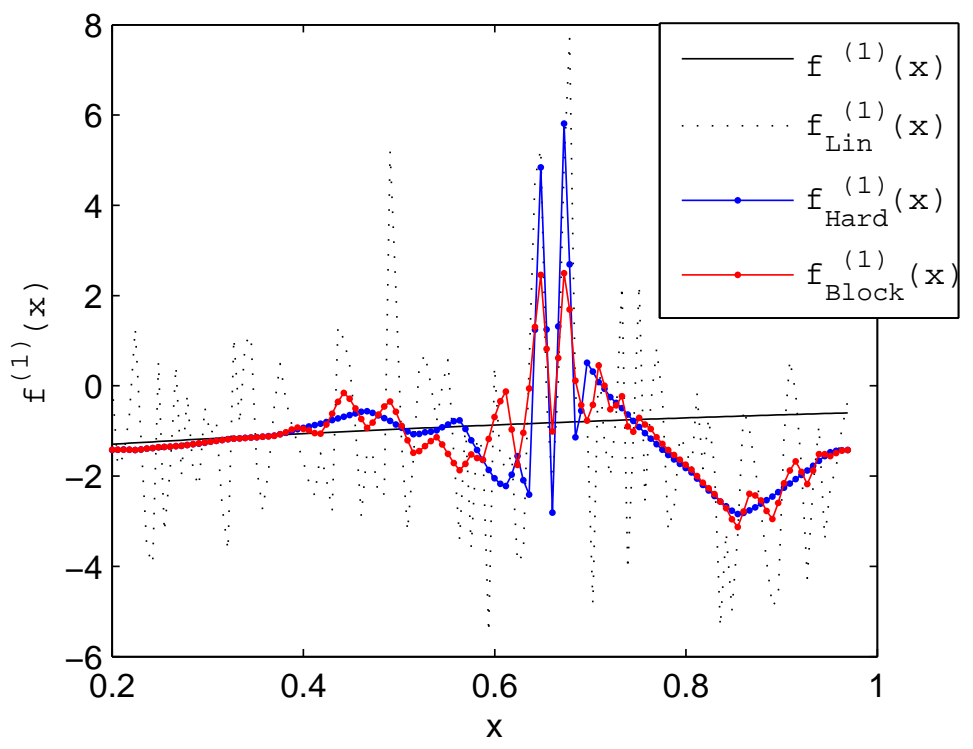

Figure 1,2. The original derivative of density function (solid line), block thresholding estimator (red line), hard thresholding estimator (blue line) and linear estimator (dotted line) respectively. 


\section{Appendix: Proof of Theorem 3.1}

First we sketch the basic steps of the proof that follows along the lines of Li (2008). First we state a version of Lemma 5.1 of $\mathrm{Li}$ (2008) for wavelet density derivatives, that compares the estimators of the wavelet coefficients to those for the complete data case. Its proof can be provided basically by mimicking the steps of the proof of $\mathrm{Li}$ (2008), hence it is omitted.

Lemma: Let $\hat{\alpha}_{j}$ and $\hat{\beta}_{i j}$ be defined as in equations (3.6) and (3.7). Also, let

$$
\begin{aligned}
\varphi_{j}^{(d)}(x) & =\phi_{j}^{(d)}(x) I(x \leq T) \quad j=0, \pm 1, \pm 2, \ldots \\
\varphi_{i j}^{(d)}(x) & =\psi_{i j}^{(d)}(x) I(x \leq T) \quad i=0,1, \ldots, R ; \quad j=0, \pm 1, \pm 2, \ldots \\
\bar{\alpha}_{j} & =\frac{(-1)^{d}}{n} \sum_{m=1}^{n} \frac{\delta_{m} \varphi_{j}^{(d)}\left(Z_{m}\right)}{1-\hat{G}\left(Z_{m}\right)}, \quad j=0, \pm 1, \pm 2, \ldots \\
\bar{\beta}_{i j} & =\frac{(-1)^{d}}{n} \sum_{m=1}^{n} \frac{\delta_{m} \varphi_{i j}^{(d)}\left(Z_{m}\right)}{1-\hat{G}\left(Z_{m}\right)}, \quad i=0,1, \ldots, R ; \quad j=0, \pm 1, \pm 2, \ldots
\end{aligned}
$$

Then the following equations hold.

$$
\begin{gathered}
\hat{\alpha}_{j}=\bar{\alpha}_{j}+\bar{W}_{j}+R_{n, j}, \quad E\left(R_{n, j}^{2}\right)=O\left(\frac{1}{n^{2}}\right) \int\left(\varphi_{j}^{(d)}\right)^{2} d F . \\
\hat{\beta}_{i j}=\bar{\beta}_{i j}+\bar{W}_{i j}+R_{n, i j}, \quad E\left(R_{n, i j}^{2}\right)=O\left(\frac{1}{n^{2}}\right) \int\left(\varphi_{i j}^{(d)}\right)^{2} d F .
\end{gathered}
$$

where

$$
\begin{aligned}
W_{j}\left(Z_{m}\right) & =U_{j}\left(Z_{m}\right)-V_{j}\left(Z_{m}\right), \quad W_{i j}\left(Z_{m}\right)=U_{i j}\left(Z_{m}\right)-V_{i j}\left(Z_{m}\right), \\
\bar{W}_{j} & =n^{-1} \sum_{m=1}^{n} W_{j}\left(Z_{m}\right), \quad \bar{W}_{i j}=n^{-1} \sum_{m=1}^{n} W_{i j}\left(Z_{m}\right),
\end{aligned}
$$




$$
\begin{aligned}
U_{j}\left(Z_{m}\right) & =\frac{(-1)^{d}\left(1-\delta_{(m)}\right)}{1-H\left(Z_{m}\right)} \int_{Z_{m}}^{\tau_{H}} \varphi_{j}^{(d)}(\omega) F(d \omega) . \\
U_{i j}\left(Z_{m}\right) & =\frac{(-1)^{d}\left(1-\delta_{(m)}\right)}{1-H\left(Z_{m}\right)} \int_{Z_{m}}^{\tau_{H}} \varphi_{i j}^{(d)}(\omega) F(d \omega) . \\
V_{j}\left(Z_{m}\right) & =(-1)^{d} \int_{-L}^{\tau_{H}} \int_{-L}^{\tau_{H}} \frac{\varphi_{j}^{(d)}(\omega) I\left(\nu<Z_{m} \wedge \omega\right)}{(1-H(\nu))(1-G(\nu))} G(d \nu) F(d \omega) . \\
V_{i j}\left(Z_{m}\right) & =(-1)^{d} \int_{-L}^{\tau_{H}} \int_{-L}^{\tau_{H}} \frac{\varphi_{i j}^{(d)}(\omega) I\left(\nu<Z_{m} \wedge \omega\right)}{(1-H(\nu))(1-G(\nu))} G(d \nu) F(d \omega) .
\end{aligned}
$$

As in Li (2008), we decompose $E\left\|\hat{f}_{1}^{(d)}-f_{1}^{(d)}\right\|_{2}^{2}$ into several parts, in view of (3.9) and (3.12):

$$
E\left\|\hat{f}_{1}^{(d)}-f_{1}^{(d)}\right\|_{2}^{2} \leq 4\left(I_{1}+I_{2}+I_{3}+I_{4}\right)
$$

where

$$
\begin{aligned}
& I_{1}=E\left\|\hat{\mathcal{K}}_{0}-\mathcal{K}_{0} f^{(d)}\right\|_{2}^{2}, \\
& I_{2}=E\left\|\sum_{i=0}^{i_{s}}\left[\sum_{k} \hat{d}_{i k} I\left(J_{i k}\right) I\left(\hat{B}_{i k}>C n^{-1}\right)-\mathcal{D}_{i} f^{(d)}\right]\right\|_{2}^{2}, \\
& I_{3}=E\left\|\sum_{i=i_{s}+1}^{R}\left[\sum_{k} \hat{d}_{i k} I\left(J_{i k}\right) I\left(\hat{B}_{i k}>C n^{-1}\right)-\mathcal{D}_{i} f^{(d)}\right]\right\|_{2}^{2}, \\
& I_{4}=E\left\|\sum_{i=R+1}^{\infty} \mathcal{D}_{i} f^{(d)}\right\|_{2}^{2},
\end{aligned}
$$

Next, we obtain the upper bounds for $I_{1}, I_{2}, I_{3}$ and $I_{4}$, as follows:

$$
\begin{aligned}
& I_{1}=O\left(n^{-1}\right) ; \\
& I_{2} \leq C\left(\log _{2} n\right)^{\frac{2-p}{p(1+2 s)}} n^{-2(s-d) /(1+2 s)} \\
& I_{3} \leq C n^{-2(s-d) /(1+2 s)} \\
& I_{4}=o\left(n^{-2(s-d) /(1+2 s)}\right),
\end{aligned}
$$

proofs of which are detailed subsequently.

Using the above bounds in Eq (4.10) the proof is completed.

Bound for $I_{1}$ : Using orthogonality of wavelets $\phi_{j}$, we have

$$
I_{1}=E \int\left(\sum_{j} \hat{\alpha}_{j} \phi_{j}-\sum_{j} \alpha_{j} \phi_{j}\right)^{2}=E \int\left(\sum_{j}\left(\hat{\alpha}_{j}-\alpha_{j}\right) \phi_{j}\right)^{2}=\sum_{j} E\left(\hat{\alpha}_{j}-\alpha_{j}\right)^{2} .
$$


From the lemma given earlier and the fact that

$$
3\left(a^{2}+b^{2}+c^{2}\right)-(a+b+c)^{2} \geq 0
$$

we have

$$
\begin{aligned}
I_{1} & \leq 3\left\{\sum_{j} E\left(\bar{\alpha}_{j}-\alpha_{j}\right)^{2}+\sum_{j} E \bar{W}_{j}^{2}+\sum_{j} E R_{n, j}^{2}\right\} \\
& =: 3\left(I_{11}+I_{12}+I_{13}\right) .
\end{aligned}
$$

Noting that

$$
\begin{aligned}
E\left(\bar{\alpha}_{j}-\alpha_{j}\right)^{2} & =E\left\{(-1)^{d} 2^{i_{0}(d+1 / 2)} n^{-1} \sum_{m=1}^{n} \frac{\delta_{m} \varphi^{(d)}\left(2^{i_{0}} Z_{m}-j\right)}{1-G\left(Z_{m}\right)}\right\}^{2}-n^{-1} \alpha_{j}^{2} \\
& =(-1)^{2 d} 2^{i_{0}(2 d+1)} n^{-1} \int\left(\varphi^{(d)}\right)^{2}(y) \frac{f_{1}^{(d)}\left((y+j) / 2^{i_{0}}\right)}{1-G\left((y+j) / 2^{i_{0}}\right)} d y-n^{-1} \alpha_{j}^{2},
\end{aligned}
$$

we obtain

$$
\sum_{j} E\left(\bar{\alpha}_{j}-\alpha_{j}\right)^{2}=2^{i_{0}(2 d+1)} n^{-1} \int\left(\varphi^{(d)}\right)^{2}(y) \sum_{j} 2^{-i_{0}} \frac{f_{1}\left((y+j) / 2^{i_{0}}\right)}{1-G\left((y+j) / 2^{i_{0}}\right)} d y-n^{-1} \sum_{j} \alpha_{j}^{2} .
$$

Since

$$
\sum_{j} 2^{-i_{0}} f_{1}\left((y+j) / 2^{i_{0}}\right) /\left(1-G\left((y+j) / 2^{i_{0}}\right)\right) \rightarrow \int f_{1} /(1-G)
$$

and

$$
\sum_{j} \alpha_{j}^{2}=O\left(\int f_{1}^{2}\right) \int\left(\varphi_{j}^{(d)}\right)^{2}
$$

we get $E \sum_{j}\left(\bar{\alpha}_{j}-\alpha_{j}\right)^{2}=2^{i_{0}(2 d+1)} n^{-1} \int f_{1} /(1-G) \int\left(\varphi^{(d)}\right)^{2}+o\left(2^{i_{0}(2 d+1)} n^{-1}\right)$, therefore

$$
I_{11}=O\left(2^{i_{0}(2 d+1)} n^{-1}\right)=O\left(n^{-1}\right) .
$$

Further from (4.7)

$$
I_{12} \leq n^{-1} \sum_{j} E W_{j}^{2}\left(Z_{1}\right) \leq 2 n^{-1} \sum_{j}\left(E U_{j}^{2}\left(Z_{1}\right)+E V_{j}^{2}\left(Z_{1}\right)\right) .
$$

In view of (4.8), applying Cauchy-Schwarz inequality and using the compact support of $\phi$, we finally obtain

$$
E U_{j}^{2}\left(Z_{1}\right) \leq \frac{1}{[(1-H(T))][(1-G(T))]} 2^{i_{0}(2 d+1)} \int\left(\varphi^{(d)}\right)^{2}(y) f_{1}^{2}\left((y+j) / 2^{i_{0}}\right) d y .
$$


Hence,

$n^{-1} \sum_{j} E U_{j}^{2}\left(Z_{1}\right)=O\left(2^{i_{0}(2 d+1)} n^{-1} \int\left(\varphi^{(d)}\right)^{2}(y) \sum_{j} 2^{-i_{0}} f_{1}^{2}\left((y+j) / 2^{i_{0}}\right) d y\right)=o\left(2^{i_{0}(2 d+1)} n^{-1}\right)$.

Similarly, we obtain

$$
E V_{j}^{2}\left(Z_{1}\right) \leq \frac{1}{[(1-H(T))]^{2}[(1-G(T))]^{2}} 2^{i_{0}(2 d+1)} \int\left(\varphi^{(d)}\right)^{2}(y) f_{1}^{2}\left((y+j) / 2^{i_{0}}\right) d y .
$$

Thus, $n^{-1} \sum_{j} E V_{j}^{2}\left(Z_{1}\right)=o\left(2^{i_{0}(2 d+1)} n^{-1}\right)$ and we get

$$
I_{12}=o\left(2^{i_{0}(2 d+1)} n^{-1}\right)=o\left(n^{-1}\right) .
$$

Using the property of $E\left(R_{n, i j}^{2}\right)$ from (4.5, we have

$$
I_{13}=O\left(n^{-2}\right) \sum_{j} \int\left(\varphi_{j}^{(d)}\right)^{2} d F=O\left(2^{i_{0}(2 d+1)} n^{-2}\right)=o\left(2^{i_{0}(2 d+1)} n^{-2}\right)=O\left(n^{-2}\right) .
$$

Now, using the bounds obtained for $I_{11}, I_{12}$ and $I_{13}$ in (4.11), we have $I_{1}=O\left(n^{-1}\right)$.

Bound for $I_{2}$ : From similar methods as for the proof of Lemma 5.6 in Li (2008), we get

$$
I_{2} \leq\left\{\sum_{i=0}^{i_{s}}\left[E \int\left(\sum_{k} \hat{d_{i k}} I\left(J_{i k}\right) I\left(\hat{B}_{i k}>C n^{-1}\right)-\mathcal{D}_{i} f^{(d)}\right)^{2} d x\right]^{1 / 2}\right\}^{2} .
$$

Writing $\mathcal{D}_{i} f^{(d)}(x)=\sum_{j} \beta_{i j} \psi_{i j}(x)=: \sum_{k} \sum_{j \in \Gamma_{i k}} \beta_{i j} \psi_{i j}(x)=: \sum_{k} d_{i k} f^{(d)}$, we have for the term in the square brackets 


$$
\begin{array}{ll}
E \int & \left(\sum_{k} \hat{d}_{i k}(x) I\left(J_{i k}\right) I\left(\hat{B}_{i k}>C n^{-1}\right)-\mathcal{D}_{i} f^{(d)}(x)\right)^{2} d x \\
\leq & 3\left\{E \int\left[\sum_{k}\left(\hat{d}_{i k}(x)-d_{i k} f^{(d)}(x)\right) I\left(J_{i k}\right) I\left(\hat{B}_{i k}>C n^{-1}\right)\right]^{2} d x\right. \\
+ & E \int_{J_{i k}}\left[\sum_{k} d_{i k} f^{(d)}(x) I\left(B_{i k}<2 C n^{-1}\right) I\left(\hat{B}_{i k} \leq C n^{-1}\right)\right]^{2} d x \\
+ & \left.E \int_{J_{i k}}\left[\sum_{k} d_{i k} f^{(d)}(x) I\left(B_{i k}>2 C n^{-1}\right) I\left(\hat{B}_{i k} \leq C n^{-1}\right)\right]^{2} d x\right\} \\
\leq & 3\left\{E \int_{\left(\hat{d}_{i k}(x)-d_{i k} f^{(d)}(x)\right)^{2} d x}\right. \\
+\quad & \left\{E \sum_{k} \int_{J_{i k}}\left(d_{i k} f^{(d)}(x)\right)^{2} d x I\left(B_{i k} \leq 2 C n^{-1}\right)\right. \\
+ & \left\{E \sum_{k} \int_{J_{i k}}\left(d_{i k} f^{(d)}(x)\right)^{2} d x I\left(B_{i k}>2 C n^{-1}\right) I\left(\hat{B}_{i k} \leq C n^{-1}\right)\right\} \\
= & 3\left(I_{21}+I_{22}+I_{23}\right),
\end{array}
$$

where $I_{22} \leq C 2^{i} n^{-1}$ and $I_{23} \leq C n^{-1}$ for all $i$. Also

$$
\begin{aligned}
I_{21} & =\sum_{j} E\left(\hat{\beta}_{i j}-\beta_{i j}\right)^{2} \\
& \leq 3\left\{\sum_{j} E\left(\bar{\beta}_{i j}-\beta_{i j}\right)^{2}+\sum_{j} E \bar{W}_{i j}^{2}+\sum_{j} E R_{n, i j}^{2}\right\} \\
& =: 3\left(I_{211}+I_{212}+I_{213}\right) .
\end{aligned}
$$

Applying the same arguments as for the term $I_{1}$, we get

$$
I_{211}=O\left(n^{-1} 2^{i(2 d+1)}\right), \quad I_{212}=o\left(n^{-1} 2^{i(2 d+1)}\right), \quad I_{213}=O\left(n^{-2} 2^{i(2 d+1)}\right) .
$$

Thus, $I_{21} \leq C 2^{i(2 d+1)} n^{-1}=O\left(n^{-\frac{2(s-d)}{1+2 s}}\right)$ and we get

$$
\begin{aligned}
I_{2} & \leq\left\{\sum_{i=0}^{i_{s}}\left[C 2^{i(2 d+1)} n^{-1}+C 2^{i} n^{-1}+C n^{-1}\right]^{1 / 2}\right\}^{2} \\
& \leq C\left\{\sum_{i=0}^{i_{s}}\left[\left(2^{i(2 d+1)} n^{-1}\right)^{1 / 2}+\left(2^{i} n^{-1}\right)^{1 / 2}+n^{-1 / 2}\right]\right\}^{2} \\
& \leq C\left(2^{i_{s}(2 d+1)} n^{-1}+2^{i_{s}} n^{-1}+i_{s}^{2} n^{-1}\right) .
\end{aligned}
$$


Now, under the assumptions of Theorem 3.1, if $p \geq 2$ and $i_{s}$ satisfies $2^{i_{s}} \simeq n^{1 /(2 s+1)}$, then $I_{2} \leq C n^{-2(s-d) /(2 s+1)}$. If $1 \leq p<2$ and $i_{s}$ satisfies $2^{i_{s}} \simeq\left(\log _{2} n\right)^{\frac{2-p}{p(1+2 s)}} n^{1 /(1+2 s)}$, then

$$
I_{2} \leq C\left(\log _{2} n\right)^{\frac{2-p}{p(1+2 s)}} n^{-2(s-d) /(1+2 s)} .
$$

Bound for $I_{3}$ : The arguments to obtain the desired bound for $I_{3}$, are similar to the proof of Lemma 5.7 in Li (2008), hence the details are omitted.

Bound for $I_{4}$ : The arguments for the term $I_{4}$ are also very similar to that for $I_{4}$ in $\mathrm{Li}$ (2008), except that the smoothing index $s$ is different. It leads to the result that for $p<2, I_{4} \leq M^{2} 2^{-2 i \sigma}$. On the basis of the choice of $R$ with $2^{R} \simeq n\left(\log _{2} n\right)^{-2}$ and $2 \sigma=1+2(s-1 / p)>2(s-d) /(1+2 s)$, we have $I_{4}=o\left(n^{-2(s-d) /(1+2 s)}\right)$. For $p \geq 2$ proof goes along the similar lines.

\section{Acknowledgements}

The authors are thankful to an anonymous referee for a careful revision of the manuscript and some constructive suggestions. Y.P. Chaubey acknowledges NSERC of Canada for partial support of this research through his discovery grant.

E. Shirazi was supported by Ordered and Spatial Data Center of Excellence of Ferdowsi University of Mashhad.

\section{References}

[1] Antoniadis, A. (2007). Wavelet methods in statistics: Some recent dvelopments and their applications. Statistics Surveys 1, 16-55.

[2] Antoniadis, A., Bigot, J. and Spatinas, T. (2001). Wavelet estimators in nonparametric regression: a comparative simulation study. Journal of Statistical Software 6. See http://www.jstatsoft.org/v06/i06.

[3] Antoniadis, A., Grégoire, G. and Nason, G. (1999). Density and hazard rate estimation for right-censored data by using wavelet methods. J. Roy. Statist. Soc. 61, 63-84.

[4] Bhattacharya, P. K. (1967). Estimation of a probability density function and its derivatives. Sankhyā A29, 373-382. 
[5] Buckheit, J.B., Chen, S., Donoho, D.L., Johnstone, I.M., and Scargle, J. (1995). About WaveLab. Technical Report USA: Department of Statistics, Stanford University. Available at : http://www-stat.standord.edu/ wavelab/.

[6] Cai, T. (1999). Adaptive wavelet estimation: A block thresholding and oracle inequality approach. Ann. Statist. 27, 898-924.

[7] Cai, T.(2002). On block thresholding in wavelet regression: Adaptivity, block size, and threshold level. Statistica Sinica 12, 1241-1273.

[8] Chaubey, Y. P., Doosti, H., Prakasa Rao, B. L. S.(2006). Wavelet based estimation of the derivatives of a density with associated variables., J Int. J. Pure Appl. Math. , 27, 97-106.

[9] Chaubey, Y. P., Doosti, H., Prakasa Rao, B. L. S. (2008). Wavelet based estimation of derivative of a density for negatively associated process. Journal of Statistical Theory and Practice, 2, 453-463.

[10] Chaubey, Y. P., Sen, A. and Sen, P. K. (2007). Smoothed funcional estimation for censored data. Encyclopedia of Statistics in Quality and Reliability. John Wiley and Sons, Chichester, UK.

[11] Chicken, E. and Cai, T. (2005). Block thresholding for density estimation: Local and global adaptivity. J. Multi. Anal. 95, 76-106.

[12] Cohen, A., Daubechies, I. and Vial, P. (1993). Wavelets on the Interval and Fast Wavelet Transforms. Applied and Computational Harmonic Analysis 27, 97-106.

[13] Dabrowska, D. M. (1995). Nonparametric Regression with Censored Covariates. J. Multi. Anal. 54, 253-283.

[14] Daubechies, I. (1988). Orthogonal bases of compactly supported wavelets. Statistica Sinica 41, 909-996.

[15] Daubechies, I. (1992). Ten Lectures on Wavelets, CBMS-NSF regional conferences series in applied mathematics. SIAM, Philadelphia.

[16] Delouille, V., Simoens, J. and von Sachs, R. (2001). Smooth design-adapted wavelets for nonparametric stochastic regression. Discussion Paper 0117, Institut de Statistique, UCL, Belgium.

[17] Devroye, L. (1987). A Course in density Estimation. Birkhaüser, MA, USA. 
[18] Diehl, S. and Stute, W. (1988). Kernel density and hazard function estimation in the presence of censoring. J. Multi. Anal. 25, 299-310.

[19] Donoho, D. L. and I.M. Johnstone, I. M. (1995). Adapting to unknown smoothness via wavelet shrinking. J. Am. Statist. Assoc. 90, 1200-1224.

[20] Donoho, D.L., Johnstone, I.M., Kerkyacharian, G. and Picard, D. (1995). Wavelet shrinkage: asymptopia? (with discussion). J. Roy. Statist. Soc. B57, 301-369.

[21] Hall, P., Kerkyacharian, G. and Picard, D. (1998). Block threshold rules for curve estimation using kernel and wavelet method. Ann. Stat. 26, 922942.

[22] Hall, P., Kerkyacharian, G. and Picard, D. (1999). On the minimax optimality of block thresholded wavelet estimators. Ann. Stat. 9, 33-50.

[23] Hall, P. and Patil, P. (1995). Formulae for mean integated squared error of nonlinear wavelet-based density estimators. Ann. Stat. 23, 905-928.

[24] Härdle, W., Kerkycharian, Picard, D. and Tsybakov, T. (1998). Wavelets, Approximations, and Statistical Applications, Lecture Notes in Statistics, 129, Springer, New York.

[25] Härdle, W., Marron, J. S. and Wand, M. P. (1990). Bandwidth Choice for Density Derivatives. J. Roy. Statist. Soc. B52, 223-232.

[26] Leblanc, F. (1996). Wavelet linear density estimator for a discrete-time stochastic process: $L_{p}-$ losses. Statist. Probab. Lett. 27, 71-84.

[27] Li, L. (2002). Hazard rate estimation for censored data by wavelet methods. Communications in Statistics - Theory and Methods 31, 943 - 960.

[28] Li, L. (2003). Non-linear wavelet-based density estimators under random censorship. J. of Statist. Plann. Inf. 117, 35-58.

[29] Li, L. (2008). On the block thresholding wavelet estimators with censored data. J. Multi. Anal. 99, 1518-1543.

[30] Li, Q. and Racine, J. S. (2006). Nonparametric Econometrics: Theory and Practice. Princeton University Press, New Jersey.

[31] Mallat, S. G. (1989). A theory for multiresolution signal decomposition: the wavelet representation. IEEE Transactions on Pattern Analysis and MAchine Intelligence, 11, 674-693. 
[32] McNichols, D. T. and Padgett, W. J. (1984). Nonparametric estimation from accelerated life tests with random censorship. Reliability Theory and Models (Charlotte, N.C., 1983), Notes Rep. Comput. Sci. Appl. Math., 10, Academic Press, Orlando, FL, 155-167.

[33] Meyer, Y. (1992). Wavelets and Operators. Cambridge University Press: Cambridge.

[34] Muller, H. and Gasser, T. (1979). Optimal convergence properties of kernel estimates of derivatives of a density function. Smoothing techniques for curve estimation. Proc. Workshop, Heidelberg, 1979, Lecture Notes in Math., 757, Springer, Berlin, 144-154.

[35] Padgett, W. J. and McNichols, D. T. (1984). Nonparametric density estimation from censored data. Commun. Stat.- Theor. and Meth. 13, 1581-1611

[36] Pagan, A. and Ullah, A. (1999). Nonparametric Econometrics. Cambridge University Press: Cambridge.

[37] Parzen, E. (1962). On estimation of a probability density function and mode. Ann. Math. Stat. 33, 1065-1076.

[38] Patil, P. (1997). Nonparametric hazard rate estimation by orthogonal wavelet methods. J. of Statist. Plann. Inf. 60, 153-168.

[39] Prakasa Rao, B. L. S. (1983). Nonparametric Functional Estimation. Academic Press: New York.

[40] Prakasa Rao, B. L. S. (1996). Nonparametric estimation of the derivatives of a density by the method of wavelets. Bull. Inform. Cyb. 28, 91-100.

[41] Rosenblatt, M. (1956). Remarks on some nonparametric estimates of a density function. Ann. Math. Stat. 27, 832-837.

[42] Silverman, B. W. (1986). Density Estimation for Statistics and Data Analysis. Chapman and Hall. New York, NY.

[43] Singh. R. S. (1977). Applications of estimators of a density and its derivatives to certain statistical problems. J. Roy. Statist. Soc. B39, 357-363.

[44] Talagrand, M. (1994). Sharper bounds for Gaussian and empirical processes, Ann. Probab. 22 (1) 2876.

[45] Tribel, H. (1992). Theory of Function Space II. Birkhä̈irkhauser Verlag, Berlin. 
[46] Vidakovic, A. (1999). Statistical Modelling by Wavelets, Springer, New York.

[47] Wand, M. P. and Jones, M. C. (1995). Kernel smoothing. Chapman and Hall, London.

[48] Wand, M. P. and Ripley, B. D. (2009). KernSmooth: Functions for Kernel Smoothing for Wand and Jones (1995). $R$ package version 2.22-19, URL http://CRAN.R-project.org/. 